\title{
VARIAÇÃO ESPAÇO-TEMPORAL DO ÍNDICE DE ÁREA FOLIAR E DO BRIX EM CANA-DE-AÇÚCAR (1)
}

\author{
MAXIMILIANO SALLES SCARPARI $\left({ }^{2}\right)$; EDGAR GOMES FERREIRA DE BEAUCLAIR $\left({ }^{3 *}\right)$
}

\begin{abstract}
RESUMO
Os objetivos do trabalho foram estudar a variação espaço-temporal do índice de área foliar (IAF) e do teor de sólidos solúveis (Brix) na cana-de-açúcar, em Piracicaba (SP), em 2003 e 2004, utilizando a geoestatística, visando melhorar a simulação da interceptação da radiação solar principalmente no ajuste do coeficiente de extinção. Verificaram-se maiores valores do IAF no ciclo de crescimento da variedade SP 80-3280 em comparação com a RB 855156 obtendo valores máximos de 6,41 e 4,27 respectivamente, medidos em 14/1/2004 com grande variação espacial nas áreas analisadas, além de maiores valores do IAF e menores do Brix em locais com maior disponibilidade hídrica, indicativo de atraso da maturação. A única medida do Brix feita em 29/4/2004 das variedades SP 80-3280 e RB 855156 indicaram valores médios de 12,86 e 17,06 respectivamente, mostrando a precocidade da RB 855156 . O uso de valores diários variáveis do coeficiente de extinção em modelos de cultura é recomendado, melhorando a simulação da interceptação da radiação solar, minimizando erros na estimativa da produtividade principalmente quando analisamos diferentes variedades de cana-de-açúcar.
\end{abstract}

Palavras-chave: coeficiente de extinção, geoestatística, modelos de cultura, planejamento.

\section{ABSTRACT \\ SPATIAL-TEMPORAL VARIATION OF LEAF AREA INDEX AND BRIX IN SUGARCANE}

The goals of this work were to study the spatial-temporal variation of leaf area index (LAI) and soluble solids (Brix) in sugarcane cultived in Piracicaba, São Paulo State, in the years of 2003 and 2004. Geoestatistic methodology was used aiming improvement in the simulation of solar radiation interception, and specially adjustment of extinction coefficient. During growth cycle larger values of leaf area index were verified in variety SP 80-3280 than in RB 85 5156. Those values ranged from 6.41 to 4.27 respectively, and were measured in $1 / 14 / 2004$, with large spatial variation. Higher values for LAI and smaller values for Brix were also observed under high water available, indicating a delay in maturation. The only Brix measurement performed in the varieties SP 80-3280 and RB 855156 on 4/29/2004 indicated average values of 12.86 and 17.06 respectively, demonstrating the precocity of RB 855156 . The use of daily variable values of the extinction coefficient is recommended, improving the simulation of solar radiation interception, minimizing mistakes estimating the productivity when different sugarcane varieties were analyzed.

Key words: extinction coefficient, geoestatistic, crop models, planning.

$\left({ }^{1}\right)$ Recebido para publicação em 26 de janeiro de 2006 e aceito em 29 de setembro de 2007.

$\left({ }^{2}\right)$ Centro Avançado de Pesquisa Tecnológica de Agronegócio de Cana, Caixa Postal 206, 14001-970 Ribeirão Preto (SP). E-mail: msscarpa@iac.sp.gov.br $\left(^{*}\right)$ Autor correspondente. Bolsista de Doutorado FAPESP.

$\left({ }^{3}\right)$ Departamento de Produção Vegetal - USP/ESALQ, Caixa Postal 9, 13418-900 Piracicaba (SP). E-mail: egfbeauc@esalq.usp.br 


\section{INTRODUÇÃO}

As folhas são responsáveis diretas pela transformação da energia solar em energia química através da fotossíntese. A fração de luz interceptada é determinada pelo índice de área foliar (IAF) sendo a área total das folhas verdes pela área de solo (WATSON, 1947).

O desenvolvimento foliar durante o ciclo da cultura pode ser relacionado com o acúmulo de grausdia e déficit hídrico (Teruel et al., 1997), e para cada estádio da cultura existem valores distintos do IAF como verificado por Leme et al. (1984), além da variação espacial existente nos ambientes de produção.

Estudando o desenvolvimento da área foliar com relação à interceptação da radiação fotossinteticamente ativa (PAR), INMAN-BAMBER (1994) verificou o fechamento da cultura sombreando as entrelinhas quase completa, quando o IAF torna-se maior que 4,0. Este fato determina a aceleração do amarelecimento e da morte das folhas, explicando que superfícies foliares maiores que 4,0 não aumentam a assimilação (Bezuidenhout, 2000). A PAR cuja faixa de comprimento de onda varia de 400 a $700 \mathrm{~nm}$ não é rotineiramente medida de acordo com BARBIERI (1993) embora esta informação seja sempre requerida para muitos propósitos incluindo a modelagem. Segundo SinClAir e Muchow (1999), a relação entre a PAR e a radiação solar é de 0,45 quando a elevação solar excede $30^{\circ}$ sobre o horizonte. Esta estimativa, entretanto, ignora a contribuição da radiação difusa que se espalha através das moléculas de gás na atmosfera e que contém uma proporção muito mais alta da PAR comparada à radiação direta. Quando a elevação solar excede $40^{\circ}$, a relação calculada da PAR somando a radiação solar direta e o componente difuso é de aproximadamente 0,60 . Combinando os componentes diretos e difusos em proporções apropriadas, a relação entre a PAR e a radiação solar está perto de 0,50 .

Geralmente, a transmissão da radiação solar no dossel de uma cultura pode ser estimada pela Lei de Beer de acordo com BARBIERI (1993). MONSI e SAEKI (1953) definiram um modelo de dossel onde cada camada é considerada uniforme, a superfície composta de folhas horizontais, opacas, sem sobreposição e a radiação decai mais ou menos exponencialmente dentro do dossel, sendo o IAF o único fator na redução da radiação. Para considerar folhas em diferentes ângulos, temos a razão entre a área de sombra e a área foliar na superfície horizontal definido como coeficiente de extinção $(k)$ (CAMPBell, 1986). O coeficiente de extinção varia com o ângulo de incidência da radiação, ou seja, com a inclinação solar, latitude, espaçamento e características foliares implicando na variação da transmissão da luz conforme relata MACHADO (1981). IINMAN-BAMBER (1994) utilizou um coeficiente de extinção variável ao longo do dia entre 0,55 e 0,58 para a variedade NCo376. BARBIERI (1993) utilizou um valor fixo de 0,58. O modelo APSIM - Sugarcane (KeAting et al., 1999) simula a fixação de carbono da atmosfera utilizando a PAR variável com a temperatura e com os teores de nitrogênio desvinculada da eficiência de transpiração. Valores fixos para coeficiente de extinção e transpiração são usados para cana-planta e socas. O índice de área foliar é relacionado com o tempo térmico e acúmulo de biomassa, sendo utilizado para o cálculo da radiação solar interceptada (O'LEARY, 2000).

Simular o dossel das plantas considerando um meio homogêneo pode trazer erros no cálculo da radiação interceptada, pois a própria natureza do dossel é heterogênea. Birch et al. (2003) apontam como solução simular o crescimento das plantas de forma individual utilizando as três dimensões do plano, considerando o desenvolvimento de órgãos como folhas e internódios individualmente, compreendendo a dinâmica de extensão vertical e expansão horizontal das partes da planta e a ocupação do espaço entre elas. Futuramente, essa simulação tridimensional individual explicará a interação entre planta daninha e cultura ou em sistemas agroflorestais, bem como será utilizada em locais onde a heterogeneidade dos solos é grande aliada a sistemas de informações geográficas (SIG). Boote e Pickering (1994) também confirmam a heterogeneidade do dossel e desse modo, a simulação da interceptação da luz usando a forma geométrica tridimensional considerando a latitude, dia do ano e horário do dia é adequada, principalmente por analisar o efeito da radiação difusa em dias ensolarados e a elevação solar.

Os objetivos do trabalho são estudar a variação espaço-temporal do índice de área foliar e do teor de sólidos solúveis em cana-de-açúcar e a conseqüente melhora na simulação da interceptação da radiação solar principalmente no ajuste do coeficiente de extinção.

\section{MATERIAL E MÉTODOS}

Este trabalho foi desenvolvido no município de Piracicaba (SP) nas seguintes coordenadas: $22^{\circ} 42^{\prime}$ Latitude $S$ e $47^{\circ} 38^{\prime}$ Longitude $\mathrm{W}$, com temperatura média anual de $21,5^{\circ} \mathrm{C}$, precipitação pluvial média anual de $1.276 \mathrm{~mm}$ e altitude média de $546 \mathrm{~m}$.

A área plantada em 24/7/2002, com a variedade SP 80-3280, possui 1,92 ha de relevo plano 
e a produtividade no primeiro corte feito em $2 / 7 / 2003$ foi de 108,22 t.ha ${ }^{-1}$; a outra área de estudo, plantada em 24/4/2002 com a variedade RB 855156 tem 3,52 ha, relevo suave ondulado e com produtividade no primeiro corte feito no dia 29/4/2003 de 83,76 t.ha ${ }^{-1}$. O espaçamento de plantio adotado nas áreas é de 1,4 m e a adubação da soqueira realizada na tríplice operação após o corte foi de $500 \mathrm{~kg}$ da formulação 4-20-20 nas duas áreas experimentais. $\mathrm{O}$ controle de invasoras se restringiu ao controle mecânico, evitando a interferência do controle químico no desenvolvimento da área foliar.

O IAF das variedades foi calculado a partir da área das folhas verdes de dez colmos seguidos na linha totalizando oito amostras georeferenciadas por análise obtida de grade de $70 \times 70 \mathrm{~m}$ em cada área experimental, com um intervalo amostral de dois meses, com início em 2/7/2003 até 15/10/2004 na variedade SP 80-3280 e de 29/4/2003 até 15/10/2004 na variedade RB 85 5156. Utilizou-se o aparelho de integração de área foliar LI-COR® model 3100 durante um ciclo da cultura e relacionado com os graus-dia (Villa Nova et al., 1972) acumulados no período (equações 1 e 2), sendo ajustados pelo método nãolinear através do programa computacional SAS "Statistical Analysis System" (SAS Institute, 1989) verificando a evolução temporal do IAF nas áreas.

Para $T m>T b$

$$
\text { GD } \quad \text { n. } \frac{T M T m}{2} \quad T b . f
$$

Para $T m \leq T b$

$$
G D \quad \text { n. } \frac{T M \quad T b^{2}}{2 T M T m} \cdot f
$$

sendo:

$$
\begin{aligned}
& f \quad N / 12 \\
& N=\text { comprimento do dia em horas, } \\
& n=\text { número de dias, } \\
& T M=\text { temperatura máxima, } \\
& T m=\text { temperatura mínima, } \\
& \mathrm{T} b=\text { temperatura base }=20^{\circ} \mathrm{C}, \text { segundo }
\end{aligned}
$$
proposto por BARBIERI et al. (1979).

As oito amostras do IAF foram georeferenciadas pelo GPS marca Garmin eTrex ${ }^{\circledR}$, gerando mapas geoestatísticos através do programa computacional $\mathrm{R}$ (Ihaka e Gentleman, 1996) e os pacotes geoR (Ribeiro Junior e Diggle, 2001), MASS (Venables e Ripley, 2002) e splancs (RowLINGSON e DigGLLE, 1993), a fim de verificar a variabilidade espacial existente nas áreas. A amostragem do teor de sólidos solúveis (Brix) seguiu a

\begin{tabular}{|c|c|c|c|c|c|c|}
\hline \multirow[b]{2}{*}{ Dias de amostragem } & \multicolumn{4}{|c|}{ IAF } & \multicolumn{2}{|c|}{ Brix } \\
\hline & $\begin{array}{c}\text { RB } 855156 \\
\text { Medido }\end{array}$ & $\begin{array}{c}\text { RB } 855156 \\
\text { Estimado modelo }\end{array}$ & $\begin{array}{c}\text { SP } 80-3280 \\
\text { Medido }\end{array}$ & $\begin{array}{c}\text { SP } 80-3280 \\
\text { Estimado modelo }\end{array}$ & RB 855156 & SP 80-3280 \\
\hline $29 / 4 / 2003$ & 0 & 0 & - & - & - & - \\
\hline $2 / 7 / 2003$ & - & - & 0 & 0 & - & - \\
\hline $20 / 8 / 2003$ & 0,68 & 0,61 & & & & \\
\hline $6 / 10 / 2003$ & 2,21 & 2,02 & 1,38 & 1,39 & - & - \\
\hline $28 / 11 / 2003$ & 3,56 & 3,92 & 4,03 & 4,36 & - & - \\
\hline $14 / 1 / 2004$ & 4,27 & 4,17 & 6,41 & 5,80 & - & - \\
\hline $3 / 3 / 2004$ & 3,77 & 3,25 & 5,35 & 5,12 & - & - \\
\hline $29 / 4 / 2004$ & 1,87 & 2,23 & 3,14 & 3,86 & 17,06 & 12,86 \\
\hline $1 / 6 / 2004$ & 1,51 & 2,07 & 2,48 & 3,63 & - & - \\
\hline $25 / 8 / 2004$ & 2 & 1,65 & 3,39 & 2,99 & - & - \\
\hline $15 / 10 / 2004$ & 1,02 & 1,03 & 3,2 & 2,01 & - & - \\
\hline$R^{2}$ & $0,94^{*}$ & $0,87^{*}$ & - & - & - & - \\
\hline Qmres & 0,1685 & 0,7931 & - & - & - & - \\
\hline
\end{tabular}
mesma metodologia utilizada para o IAF sendo feita somente em 29/4/2004 utilizando o refratômetro de campo gerando o mapa geoestatístico do início da maturação das variedades na safra 2004/2005. Na tabela 1, apresentam-se os valores de IAF e Brix nas respectivas datas de coleta.

Tabela 1. Valores médios do IAF e do Brix nas respectivas datas de coleta 
O solo é classificado como Cambissolo Háplico textura média, de acordo com a Empresa Brasileira de Pesquisa Agropecuária - EMBRAPa (1999) tendo a seguinte análise química realizada em 3/7/ 2003 na área da variedade SP 80-3280, em profundidade de 0 a $25 \mathrm{~cm}: \mathrm{pH}\left(\mathrm{CaCl}_{2}\right)=5,2 ;$ M.O. $\left(\right.$ g. $\left.\mathrm{dm}^{-3}\right)=5,2 ; \mathrm{P}_{\text {resina }}\left(\mathrm{mg} \mathrm{dm}^{-3}\right)=128 ; \mathrm{Ca}\left(\mathrm{mmol} \mathrm{dm}^{-}\right.$ $\left.{ }^{3}\right)=82 ; \mathrm{Mg}\left(\mathrm{mmol} \mathrm{dm}^{-3}\right)=40 ; \mathrm{K}\left(\mathrm{mmol} \mathrm{dm}^{-3}\right)=3,2$; $\mathrm{H}+\mathrm{Al}\left(\mathrm{mmol} \mathrm{dm}^{-3}\right)=47 ; \mathrm{CTC}\left(\mathrm{mmol} \mathrm{dm}^{-3}\right)=172 ; \mathrm{V} \%$ $=72 \mathrm{e} \mathrm{M} \%=0$ e em 3/5/2003 a análise de solo na profundidade de 0 a $25 \mathrm{~cm}$ foi feita na área da variedade RB 855156 tendo como resultado: $\mathrm{pH}$ $\left(\mathrm{CaCl}_{2}\right)=4,6$; M.O. $\left(\mathrm{g} \cdot \mathrm{dm}^{-3}\right)=2,6 ; \mathrm{P}_{\text {resina }}\left(\mathrm{mg} \mathrm{dm}^{-3}\right)=$ 13; $\mathrm{Ca}\left(\mathrm{mmol} . \mathrm{dm}^{-3}\right)=46 ; \mathrm{Mg}\left(\mathrm{mmol}^{-3} \mathrm{dm}^{-3}\right)=30 ; \mathrm{K}$ $\left(\mathrm{mmol} \mathrm{dm}{ }^{-3}\right)=2,9 ; \mathrm{H}+\mathrm{Al}\left(\mathrm{mmol} \mathrm{dm}{ }^{-3}\right)=66 ; \mathrm{CTC}(\mathrm{mmol}$ $\left.\mathrm{dm}^{-3}\right)=145 ; \mathrm{V} \%=54$ e $\mathrm{M} \%=13$.

Para o ajuste da evolução temporal do IAF utilizou-se a equação apresentada por TERUEL et al. (1997), cujos parâmetros foram obtidos pelo procedimento não linear do SAS utilizando o método de Gauss-Newton.

$$
\begin{aligned}
& I A F_{S P 80} 3280 \quad e^{14,9457 * G D^{3,0187} * e^{0,00439 * G D}} \\
& I A F_{R B 855156} e^{16,31 * G D^{3,2149} * e^{0,00471^{*} G D}}
\end{aligned}
$$

Sabendo o valor correto do coeficiente de extinção $(k)$ de cada variedade, pode-se estimar o valor máximo do IAF, considerando a interceptação de $95 \%$ da PAR incidente no topo do dossel sendo:

$$
I A F=\ln \left(I o / I^{*} I o\right) / k
$$

Em que:

$$
\ln =\log \text { neperiano, }
$$

$I o=$ radiação solar incidente no topo do dossel (cal.cm $\left.{ }^{-2} \cdot \mathrm{min}\right)$,

$I=$ radiação transmitida após atravessar um dado índice de área foliar (cal.cm ${ }^{-2}$.min),

$$
k=\text { coeficiente de extinção. }
$$

Pela equação (6), estimamos o valor máximo do IAF, considerando a interceptação de $95 \%$ da PAR incidente no topo do dossel. Como exemplo, utilizouse $k=0,58$ (BARBIERI, 1993; IBMAN-BAMBER, 1994).

$$
I A F=\ln \left(I o / I^{*} I o\right) / k=\ln \left[0,95 /\left(0,05^{*} 0,95\right)\right] / 0,58=
$$

\section{5,16}

Como indicador da precisão do ajuste dos modelos não-lineares do IAF, utilizou-se o parâmetro denominado de coeficiente de determinação $\left(R^{2}\right)$ calculado entre os valores medidos e estimados pelo modelo além do quadrado médio do resíduo (Qmres).

\section{RESULTADOS E DISCUSSÃO}

As áreas foram escolhidas por representarem boa parte das áreas próprias da usina sendo classificada como ambiente D2 (Prado et al., 2002). Segundo Prado et al. (2002), ambientes D2 incluem solos mesoálicos e argilosos com baixa disponibilidade hídrica. Além disso, poucas áreas na usina possuem duas variedades distintas em termos de época de maturação plantadas próximas, pois o planejamento é feito no intuito de cortar todo o setor de uma só vez, evitando o retorno das frentes de corte, carregamento e transporte na mesma área. Apesar de próximas, observa-se maior declividade na área da variedade RB 85 5156, menos fértil, sendo possível que uma fração do adubo aplicado seja levada pela enxurrada até a área da SP 80-3280, tornando-a, ao longo do tempo, mais fértil, já que o manejo é semelhante em ambas as áreas.

A variação espaço-temporal do IAF das áreas pode ser vista na Figuras 1 e 2. Apresentou-se somente uma análise da variação espacial do IAF e do Brix realizada em 29/4/2004 por representar o início da safra 2004/ 2005 (Figuras 2 e 3). Com relação à distribuição espacial dos atributos, a geoestatística possibilita a partir dos dados amostrados estimar valores a pontos intermediários utilizando as técnicas do semivariograma e da interpolação. Semivariograma é o modelo da variação espacial dos dados considerando a semivariância, ilustrando a relação entre a variância das amostras e suas distâncias. Considerando a relação entre as amostras é possível estimar a distância máxima em que há dependência espacial (Mulla, 1993). Submetendo os dados para a análise fez-se a verificação da existência da dependência espacial e o ajuste exponencial do semivariograma pelo método dos mínimos quadrados e posterior interpolação utilizando a krigagem simples. A krigagem simples é o procedimento que permite interpolar dados para uma determinada configuração, visto que a média é estatisticamente constante em toda a área experimental.

$\mathrm{Na}$ área da variedade RB 855156 (Figura 2) observa-se claramente maior IAF na parte médio-baixa da área onde ocorre maior acúmulo de água e desse modo um excesso hídrico. Por outro lado, na parte alta pode haver maior déficit hídrico, e a cana-deaçúcar ter menor rendimento em colmos, entrando em maturação mais precocemente que na parte baixa (SCARPARI, 2007; Scarpari e Beauclair, 2004), confirmado através dos maiores valores de Brix na parte alta pela figura 3. Na figura 2, na área da variedade SP 80-3280, a variabilidade espacial do IAF é menor, verificando-se que em áreas mais planas este fator varia pouco, tendendo o IAF ser maior nas extremidades onde a área é mais baixa e há maior acúmulo de água. 
IAF

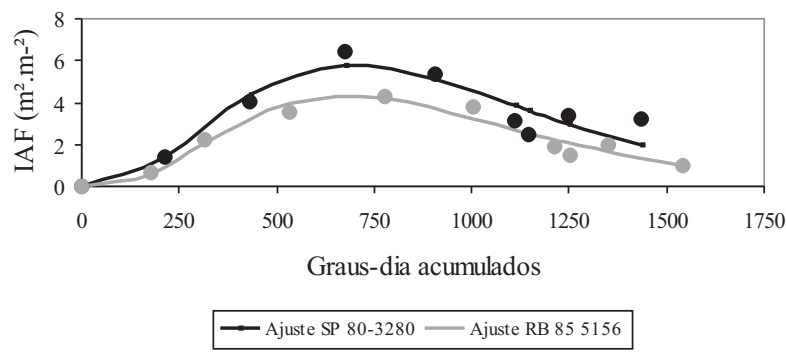

Figura 1. Evolução temporal do IAF medido em 2003 e 2004.

Analisando a evolução temporal do IAF (Figura 1 e Tabela 1), os maiores valores do IAF são observados na variedade SP 80-3280 em todo o período da amostragem, porém o maior erro no ajuste do modelo não-linear do IAF também foi notado nesta variedade, em comparação com a RB 85 5156, com um valor do quadrado médio do resíduo de 0,7931 e um valor do $R^{2}$ $=0,87^{*}$. A vantagem do maior IAF está no fato de a área foliar individual ser maior já que o número de folhas verdes é semelhante nas duas variedades. Isto explica a razão de a SP 80-3280 ter rápido fechamento da entrelinha com melhor desenvolvimento do dossel e maior interceptação da radiação solar. Observações semelhantes foram feitas por Sinclair et al. (2004) estudando algumas variedades de Canal Point onde o número de folhas verdes era semelhante, entretanto a área foliar individual variava. Na variedade RB 855156 observaram-se menores valores do IAF (Tabela 1), entretanto melhor ajuste do modelo não-linear por ocorrer valor menor do quadrado médio do resíduo $(0,1685)$ e maior valor do $R^{2}=0,94^{*}$.

Quando o IAF atinge o valor máximo obtido na equação (7) é denominado de IAF crítico (RHODES, 1973) e apenas $5 \%$ da luz incidente atingem o solo. Com o resultado obtido na equação (7), verificamos que o valor de $k=0,58$ pode ser aplicado perfeitamente na modelagem da interceptação da radiação solar na variedade RB 85 5156, entretanto este valor é alto para a variedade SP 80-3280 por apresentar um IAF maior que 5,16. Utilizando o máximo valor do IAF obtido na amostragem para a variedade SP 80-3280 que é 6,41, temse um valor empírico de $k=0,467$ indicativo de folha mais vertical e melhor aproveitamento da luz nas folhas baixeiras. Observações de campo indicam que o coeficiente de extinção também varia com o desenvolvimento; inicialmente um valor baixo em torno de 0,47 e próximo ao pico do IAF, um valor de 0,58 (ZHOU et al., 2003). Em função da elevação solar e do ângulo foliar, GOUDRIAAN (1988) usou uma aproximação para o cálculo diário da variação do coeficiente de extinção. Assim, modelos de cultura devem considerar tanto a variação temporal como a espacial do IAF na simulação da interceptação da radiação solar, além de distintos coeficientes de extinção, minimizando desta forma erros na estimativa da produtividade.

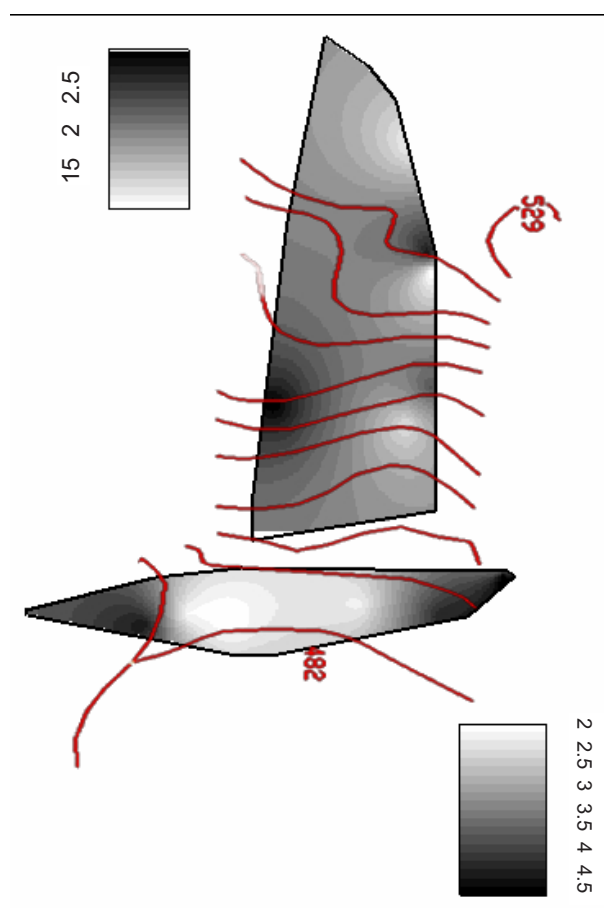

Figura 2. Variabilidade espacial do IAF das variedades RB 855156 (área maior = 3,52 ha) e SP 80-3280 (área menor $=1,92$ ha) avaliada em 29/4/2004 com as respectivas legendas ao lado das áreas.

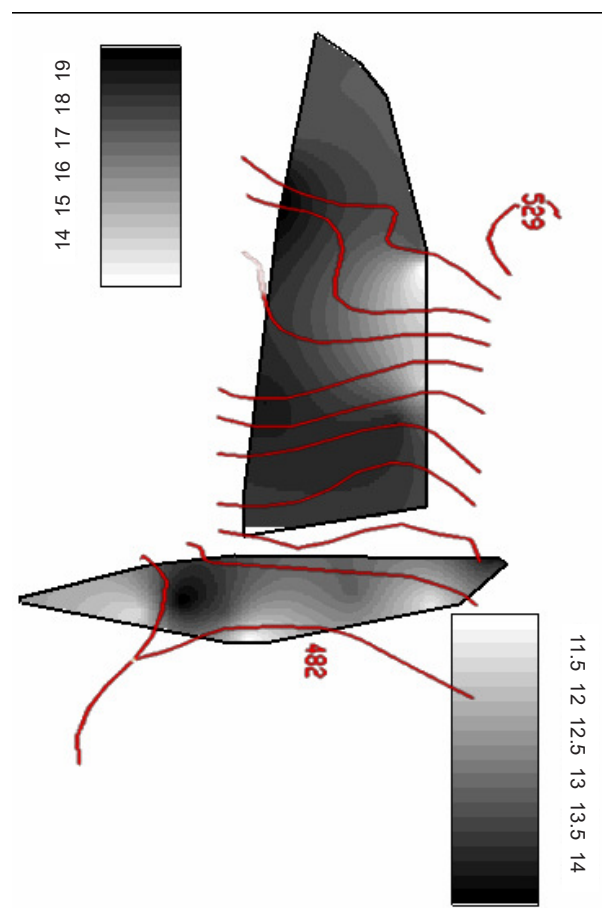

Figura 3. Variabilidade espacial do Brix das variedades RB 855156 (área maior = 3,52) e SP 80-3280 (área menor $=1,92$ ) avaliada em $29 / 4 / 2004 \mathrm{com}$ as respectivas legendas ao lado das áreas. 


\section{CONCLUSÕES}

1. Existe grande variabilidade espacial e temporal do índice de área foliar devendo ser considerada na modelagem da interceptação da radiação solar para cana-de-açúcar, buscando o melhor modelo que se ajusta aos valores medidos do índice de área foliar.

2. O uso diário de valores variáveis do coeficiente de extinção em modelos de cultura é recomendado melhorando a simulação da interceptação da radiação solar, minimizando erros na estimativa da produtividade, principalmente, quando analisamos diferentes variedades de cana-deaçúcar.

\section{REFERÊNCIAS}

BARBIERI, V. Condicionamento climático da produtividade potencial da cana-de-açúcar (Saccharum spp.): Um modelo matemático-fisiológico de estimativa. 1993. 140p. Tese (Doutorado) - Escola Superior de Agricultura “Luiz de Queiroz", Universidade de São Paulo, Piracicaba.

BARBIERI, V.; BACCHI, O.O.S.; VILLA NOVA, N.A. Análise do fator temperatura média do ar no desenvolvimento da cana-de-açúcar (Saccharum spp.). CONGRESSO BRASILEIRO DE AGROMETEOROLOGIA, 1., Mossoró, 1979. Anais... Mossoró, 1979. p.192-197.

BEZUIDENHOUT, C.N. A model review and proposed mechanistic tiller model for the CANEGRO sugarcane crop model. 2000. 78p. M.Tech. thesis - Technikon Natal, Durban.

BIRCH, C.J.; ANDRIEU, B.; FOURNIER, C.; VOS, J.; ROOM, P. Modelling kinetics of plant canopy architecture-concepts and applications. European Journal of Agronomy, v.19, p.519-533, 2003.

BOOTE, K.J.; PICKERING, N.B. Modeling photosynthesis of row crop canopies. HortScience, Alexandria, v.29, p.1423-1434, 1994.

CAMPBELL, G.S. Extinction coefficients for radiation in plant canopies calculated using an ellipsoidal inclination angle distribution. Agricultural and Forest Meteorology, Amsterdam, v.36, p.317-321, 1986.

EMPRESA BRASILEIRA DE PESQUISA AGROPECUÁRIA. Sistema Brasileiro de Classificação de Solos. Brasília, 1999. 412p.

GOUDRIAAN, J. The bare bones of leaf-angle distribution in radiation models for canopy photosynthesis and energy exchange. Agricultural and Forest Meteorology, Amsterdam, v.43, p.155-169, 1988.

IHAKA, R.; GENTLEMAN, R. R: A Language for Data Analysis and Graphics. Journal of Computational and Graphical Statistics, v.5, p.299-314, 1996.
INMAN-BAMBER, N.G. Temperature and seasonal effects on canopy development and light interception of sugarcane. Field Crops Research, Amsterdam, v.36, n.1, p.41-51, 1994.

KEATING, B.A.; ROBERTSON, M.J.; MUCHOW, R.C.; HUTH, N.I. Modelling sugarcane production systems. 1. Development and performance of the sugarcane module. Field Crops Research, Amsterdam, v.61, n.3, p.253-271, 1999.

LEME, E.J.A.; MANIERO, M.A.;GUIDOLIN, J.C. Estimativa da área foliar da cana-de-açúcar. Cadernos Planalsucar, n.2, p.3-9, 1984.

MACHADO, E.C. Um modelo matemático-fisiológico para simular o acúmulo de matéria-seca na cultura da cana-deaçúcar (Saccharum spp.). 1981. 115p. Dissertação (Mestrado) Instituto de Biologia/UNICAMP, Campinas.

MONSI, M.; SAEKI, T. Uber den lichtfacktor in den pflanzengesell-schaften und seine bedeutung fur die Stoffproduktion. Japanese Journal of Botany, Tokio, v.14, p.22-52, 1953.

MULLA, D.J. Geoestatistic, remote sensing and precision farming. In: PIERCE, F.J.; SADLER, E.J. (Ed.) Precision agriculture: spatial and temporal variability of environmental quality. New York: John Wiley \& Sons, 1997. p.100-119.

O'LEARY, G.J. A review of three sugarcane simulation models with respect to their prediction of sucrose yield. Field Crops Research, Amsterdam, v.68, p.97-111, 2000.

PRADO, H.; LANDELL, M.G.A.; ROSSETTO, R. A importância do conhecimento pedológico nos ambientes de produção de cana-de-açúcar. In: REUNIÃO BRASILEIRA DE MANEJO E CONSERVAÇÃO DE SOLO E ÁGUA., 14., Cuiabá, 2002. Anais... Cuiabá: SBCS, 2002. CD-ROM.

RHODES, I. Relationship between canopy structure and productivity in herbage grasses and its implications for plant breeding. Herbage Abstracts, v.43, p.129-133, 1973.

RIBEIRO Jr., P.J.; DIGGLE, P.J. geoR: A package for geostatistical analysis. R-NEWS, v.1, p.15-18, 2001.

ROWLINGSON, B.; DIGGLE, P. Splancs: spatial point pattern analysis code in S-Plus. Computers and Geosciences, Amsterdam, v.19, p.627-655, 1993.

SAS INSTITUTE. SAS Language and procedures: Usage, Version 6. Cary, NC: Sas Institute, 1989. 638p.

SCARPARI, M.S. PREDPOL: Um modelo de previsão da maturação da cana-de-açúcar visando planejamento otimizado. 2007. 120p. Tese (Doutorado em Fitotecnia) - Escola Superior de Agricultura "Luiz de Queiroz", Universidade de São Paulo, Piracicaba.

SCARPARI, M.S.; BEAUCLAIR, E.G.F. de Sugarcane maturity estimation through edaphic-climatic parameters. Scientia Agricola, Piracicaba, v.61, n.5, p.486-491, 2004.

SINCLAIR, T.R.; GILBERT, R.A.; PERDOMO, R.E.; SHINE JR., J.M.; POWELL, G. MONTES, G. Sugarcane leaf area development under field conditions in Florida, USA. Field Crops Research, Amsterdam, v.88, p.171-178, 2004. 
SINCLAIR, T.R.; MUCHOW, R.C. Radiation use efficiency. Advances in Agronomy, San Diego, v.65, p.215-265, 1999.

TERUEL, D.A.; BARBIERI, V.;FERRAROJUNIOR, L. A. Sugarcane leaf area index modeling under different soil water conditions. Scientia Agricola, Piracicaba, v.54, n.esp., p.39-44, 1997.

VENABLES, W.N.; RIPLEY, B.D. Modern Applied Statistics with S. New York: Springer, 2002. 512p.

VILLA NOVA, N.A.; PEDRO JÚNIOR, M.J.; PEREIRA, A.R.; OMETTO, J.C. Estimativa de graus-dia acumulados acima de qualquer temperatura base, em função das temperaturas máxima e mínima. Caderno de Ciência da Terra, v.30, n.2, p.1-8, 1972.
WATSON, D.J. Comparative physiological studies on growth of field crops. I. Variation in net assimilation rate and leaf area between species and varieties, and within and between years. Annals of Botany, Oxford, v.11, p.41-76, 1947.

ZHOU, M.; SINGELS, A.; SMIT, M. Physiological parameters for modelling varietal differences in sugarcane canopy development in the south east lowveld of Zimbabwe. Proceedings of the South African Sugarcane Technologists' Association, 77, p.32-40, 2003. 